\title{
Molecular

\section{Predicting most probable conformations of a given peptide sequence in the random coil state}

\author{
Cigdem Sevim Bayrak and Burak Erman* \\ Received 8th May 2012, Accepted 3rd August 2012 \\ DOI: $10.1039 / \mathrm{c} 2 \mathrm{mb} 25181 \mathrm{~g}$
}

In this work, we present a computational scheme for finding high probability conformations of peptides. The scheme calculates the probability of a given conformation of the given peptide sequence using the probability distribution of torsion states. Dependence of the states of a residue on the states of its first neighbors along the chain is considered. Prior probabilities of torsion states are obtained from a coil library. Posterior probabilities are calculated by the matrix multiplication Rotational Isomeric States Model of polymer theory. The conformation of a peptide with highest probability is determined by using a hidden Markov model Viterbi algorithm. First, the probability distribution of the torsion states of the residues is obtained. Using the highest probability torsion state, one can generate, step by step, states with lower probabilities. To validate the method, the highest probability state of residues in a given sequence is calculated and compared with probabilities obtained from the Coil Databank. Predictions based on the method are $32 \%$ better than predictions based on the most probable states of residues. The ensemble of " $n$ " high probability conformations of a given protein is also determined using the Viterbi algorithm with multistep backtracking.

\section{Introduction}

Backbone conformations of a protein can be described by $\varphi-\psi$ torsion angles of the amino acids that specify the rotational freedom. It is already known that some values of torsion angles are more populated than the others on the Ramachandran map and these preferences are different for different types of amino acids. ${ }^{1}$ Furthermore, preferences for a given residue are dependent on the states of the neighboring residues. ${ }^{2-10}$ In this work, the problem of predicting the high probability conformations of a protein using the rotational preferences obtained by knowledge-based approaches is discussed. Neighbor dependence is taken into consideration both in constructing the probabilities and in the generation of conformations.

Most of the computational work in predicting peptide conformations has been confined to secondary structure prediction for the native state. In the present work, we do not restrict our study to the determination of secondary structure. We specifically focus on determining the high probability conformations of peptides in the random coil state. For this reason, we use a coil library ${ }^{11}$ for determining the probabilities. Use of a secondary structure library would be more appropriate for the secondary structure prediction of a given sequence, which we do not pursue here.

Computational Science and Engineering Program, Koc University, 34450, Sariyer, Istanbul,Turkey.E-mail:berman@ku.edu.tr;

Fax: +90212338 1548; Tel: +902123381704
The torsion state of a residue is defined by a region on the Ramachandran map and these regions are separated by energy barriers. Existing studies on torsion angle predictions use a different number of states with different definitions. For example, Bystroff et al., took eleven states covering the full Ramachandran map in their hidden Markov model for local sequence-patterns in proteins (HMMSTR). ${ }^{12}$ Kuang et al. ${ }^{13}$ assumed a smaller number of states each of which correspond to a large number of states. They used four major torsion states occupying $80 \%$ of the Ramachandran map and achieved $77.3 \%$ accuracy in predicting torsion angles using SVM methods. Zimmermann and Hansmann emphasized that the torsion state prediction would provide more structural information than secondary structure predictions since the secondary structure has a complex definition. ${ }^{14}$ Estimated from the work of Lovell et al., ${ }^{15}$ they introduced ten regions. However, due to few number of samples, they based their results on three regions (right handed alpha helix, beta strand, and outside of these regions). The achieved 3 -state accuracy is around $82 \%$ using SVMs. Xin et al. presented two probabilistic methods (MEMM and CRF) to predict torsion angles using sequence profiles of residues and used fifteen regions proposed by Shortle that covers $43 \%$ of the Ramachandran map to define different coarse-grained classes. ${ }^{16}$ The achieved prediction accuracies (that is below 70\%) of each class are given. ${ }^{17}$

Previous studies all provide the prediction of the most probable torsion state for each residue and do not consider a probability distribution. However, instead of predicting only the most probable torsion state or the secondary structure, 
predicting the probability distribution of the states or secondary structures is an approach that may be useful for sampling the conformational space and may provide a detailed analysis of the space. ${ }^{18-20}$ Along this line, Helles and Fonseca proposed an analysis of probability distribution of torsion angles of coil residues using neural networks. ${ }^{18}$ They calculated the distribution of dihedral angles for $30^{\circ} \times 30^{\circ}$ bins and showed that the prediction accuracy increases as the number of considered bins increases. Helles and Fonseca showed that the neural network model they adopted outperforms the basic statistical method that simply predicts the most populated bin in the database.

In this work, our aim is to find a probability distribution of the torsion states of a given peptide sequence in the random coil state. We adopt the Rotational Isomeric States (RIS) formalism from polymer theory ${ }^{21}$ for calculating the probability distribution. Here, we identify eleven torsion states following the work of Karplus $^{22}$ that indicates the distinct regions not only for helices or strands but also for loops, beta bridges, etc. (see the notation in ref. 22-24). This knowledge provides an efficient conformational sampling. We derive the probability distribution based on a Hidden Markov Model (HMM) and the Viterbi algorithm. Dependence of the conformational probability of a given residue on the type and conformation of its neighbors is taken into consideration in constructing the probabilities, in the RIS calculations and in the Viterbi algorithm as will be seen in the rest of the paper. Our aim is similar to that of Helles and Fonseca. We depart in the method of calculations, where their approach is based on a neural network and ours on the generation of the partition function of a chain by the matrix multiplication scheme. Our ultimate aim, however, is to determine the most probable conformation and an ensemble of conformations that are of high probability. Recently, ensembles of intrinsically disordered proteins (IDP) have been constructed by Daughdrill et al. ${ }^{25}$ which is similar to our approach in spirit but different in methodology, where our high probability conformations are generated using an appropriate library, the RIS scheme, a Hidden Markov Model and the Viterbi algorithm with multistep backtracking. ${ }^{26}$ As we explain in detail in the following section, we base the present calculations on a coil library. However, choice of an IDP library would clearly lead to the generation of high probability conformations of IDP's, which are known to have statistical differences than random coils as pointed out by SchweitzerStenner ${ }^{27}$ in the recent special issue of Molecular Biosystems on Intrinsically Disordered Proteins. ${ }^{28}$

The number of occurrences of the residues in the databank in the defined states leads to the corresponding probabilities. The determination of high probability conformations of a given peptide sequence of length $N$ is a computationally difficult problem. Considering a state space of eleven torsion states leads to $11^{N}$ possible conformations from which one has to choose the optimum one. The Markov assumption and the use of the Viterbi algorithm simplify the problem significantly.

\section{Methods and materials}

\section{Knowledge based database}

Since we consider the conformations as random denatured forms and since the coil library stores the fragments from the

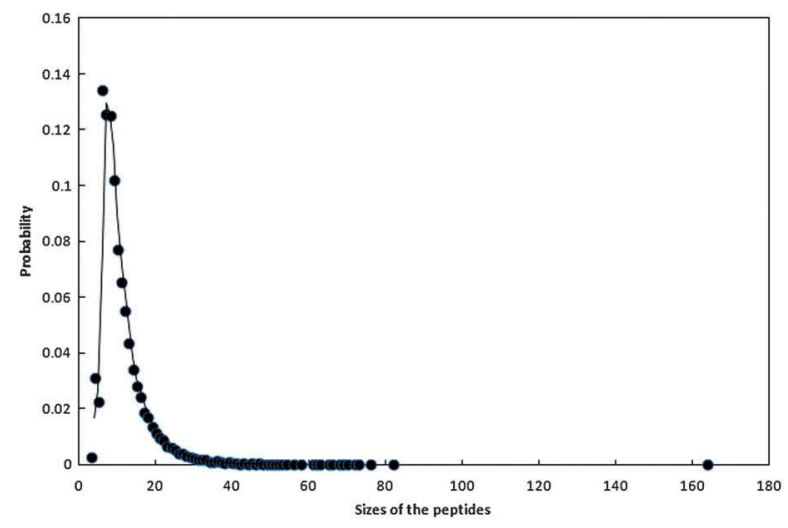

Fig. 1 Distribution of the sizes of the peptides in the coil library. Total number of peptides is 24057 .

disordered conformations that are neither alpha-helix nor beta-strand, the coil library is a good source for calculation of the probabilities. ${ }^{11}$ We used the coil library given by the Rose group http://www.roselab.jhu.edu/coil/, September 2011 version. The library contains fragments that have less than $20 \%$ sequence identity, better than 1.6 Angstrom resolutions and a refinement factor of 0.25 or better and contains 24112 fragments extracted from the Protein Data Bank. The library contains segments obtained after removal of secondary structures, the segments that are classified as alpha helix or beta strand and the one-residue coils. We remove the chains including UNK or ASX types of residues or the chains with less than 3 residues. The remaining set contains 261548 residues. The fragment size distribution is given in Fig. 1. The probabilities depend on the choice of the state space. Since the coil library is a good representative set of proteins in the coiled state and our interest is the coiled conformations of proteins, occurrence probabilities of residues in torsion states are calculated over the coil library.

\section{Torsion states}

We use the eleven torsion states introduced by Karplus. ${ }^{22}$ The descriptions of the states are given in Table 1.

\section{Methods}

It has been previously shown that the torsion preferences of a residue depend on the type and state of neighbouring residues. ${ }^{6,8}$ In Fig. 2, the left neighbor dependence of arginine is shown as an example. The frequencies are plotted in the $30^{\circ} \times 30^{\circ}$ regions of the Ramachandran map. Each $30^{\circ} \psi$ region

Table 1 Description of the twelve torsion states

State $1 \quad \varepsilon^{\prime} \quad$ Mirror image of the extended region $\varepsilon$

State $2 \varepsilon \quad$ The extensive regions, $\varphi>0, \psi \approx \pm 180^{\circ}$

State $3 \quad \alpha_{\mathrm{R}}$ Right-handed alpha helix

State $4 \quad \gamma \quad$ Tight turn region

State $5 \quad \delta_{\mathrm{R}}$ The right handed bridge region between two $\beta$-strands

$\begin{array}{llll}\text { State } 6 & \delta_{\mathrm{L}} & \text { Mirror image of the } \delta_{\mathrm{R}} \text { region }\end{array}$

State $7 \quad \zeta \quad$ Region observed mostly in residues preceding Pro

State $8 \quad \gamma^{\prime}$ Inverse tight region

State $9 \quad \alpha_{L} \quad$ Mirror image of $\alpha_{R}$

State $10 \quad \beta_{\mathrm{S}}$ Extended beta sheet forming region

State $11 \beta_{\mathrm{P}}$ Region with extended polyproline-like helices 

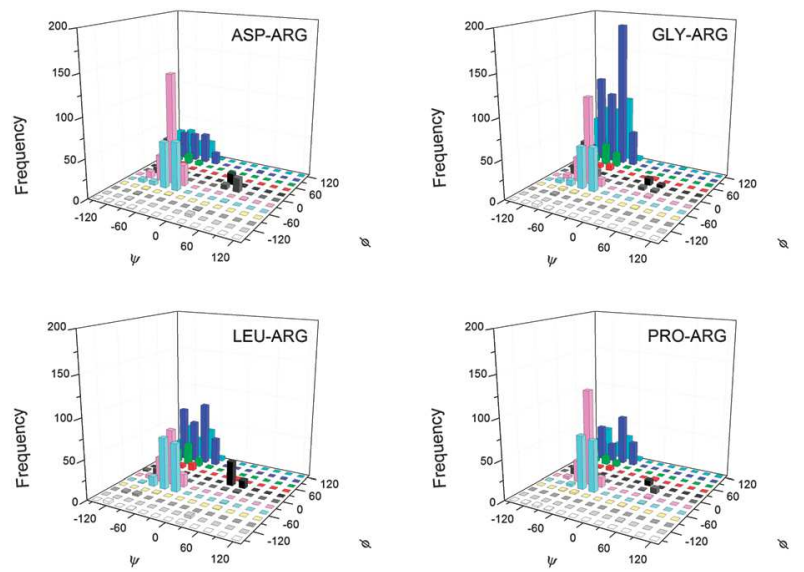

Fig. 2 Arginine has different $\Phi-\Psi$ distributions depending on the previous near neighbor.

is shown in a different color in order to increase the clarity. For certain pairs, neighbor dependence of state probabilities is significant and is included in our calculations. We treat the problem of the prediction of a torsion state as a Markov process and consider the correlations between the near neighbor residues.

In the following sections, we first define the Hidden Markov model of the problem and then we provide the calculation of the parameters of the HMM in detail. We then introduce the Viterbi algorithm for searching the state sequence that has the maximum probability of occurrence. The probability distribution of the torsion states of the residues is determined using the Viterbi algorithm. Lastly, we define a multistep backtracking algorithm that is used to guess the high probability conformations.

\section{Hidden Markov model}

In a hidden Markov model there is an observed sequence of emitted symbols which is defined by $\mathrm{O}=\mathrm{O}_{1}, \mathrm{O}_{2}, \mathrm{O}_{3}, \ldots$ and a sequence of states visited which is defined by $Q=q_{1}, q_{2}$, $\mathrm{q}_{3}, \ldots$ Further information on Hidden Markov models can be found in ref. 23 and 26. The observed sequence of our model is the given peptide sequence and the torsion state sequence is the hidden state sequence to be determined. The definitions of components of the model are given below:

1. $A=a_{1}, a_{2}, \ldots, a_{20}$, the set of 20 amino acids

2. $\mathrm{S}=\mathrm{s}_{1}, \mathrm{~s}_{2}, \ldots \mathrm{s}_{11}$, the set of 11 torsion states

3. $\boldsymbol{P}=\left(p_{i j}\right)$, the transition probability matrix where $p_{i j}=$ $\boldsymbol{P}\left(\mathrm{q}_{t+1}=\mathrm{S}_{j} \mid \mathrm{q}_{t}=\mathrm{S}_{i}\right)$ that is the probability of $t+1$ st amino acid is in state $\mathrm{S}_{j}$ given that $t$ th amino acid is in the state $\mathrm{S}_{i}$.

4. $b_{i}(\mathrm{a})=\boldsymbol{P}\left(\mathrm{S}_{i}\right.$ emits a symbol a $)$. The emission probabilities that are the probability of state $S_{i}$ emits amino acid a.

5. $\boldsymbol{\Pi}=\left(\pi_{i}\right)$. An initial distribution vector where $\pi_{i}=$ $\boldsymbol{P}\left(\mathrm{q}_{1}=\mathrm{S}_{i}\right)$

\section{Evaluation of a priori probabilities}

Here we adopt the transition probabilities for each dipeptide of the given sequence and the emission probabilities of each residue from the coil library.
The pair wise dependent probabilities $P_{X Y}\left(\mathrm{~S}_{i}, \mathrm{~S}_{\mathrm{j}}\right)$, where $\mathrm{S}_{i}$ is a torsion state defined by the two torsion angles $(\varphi, \psi)$ and $i, j=1,2, \ldots, 11$ are determined according to

$$
P_{X Y}\left(\mathrm{~S}_{i} \mathrm{~S}_{j}\right)=\frac{N_{X Y}\left(\mathrm{~S}_{i} \mathrm{~S}_{j}\right)}{\sum N_{X Y}}
$$

Here, $N_{X Y}\left(\mathrm{~S}_{i}, \mathrm{~S}_{j}\right)$ indicates the number of residue pairs observed having the indicated values of the argument. The term $\Sigma N_{X Y}$ in the denominator is the total number of observed dipeptides of $X Y$ in all possible states. The probabilities calculated in this manner are the a priori probabilities; because the conditionality of the dipeptide under consideration that being embedded into the given specific amino acid sequence of the protein has not been used.

We define a conformational energy for a given residue $Y$ in the dipeptide $X Y$ along the primary sequence of the protein as

$$
E_{X Y}\left(\mathrm{~S}_{i} \mathrm{~S}_{j}\right)=-R T \ln \left(\frac{P_{X Y}\left(\mathrm{~S}_{i} \mathrm{~S}_{j}\right)}{P_{X}^{0}\left(\mathrm{~S}_{i}\right) P_{Y}^{0}\left(\mathrm{~S}_{j}\right)}\right)
$$

where the superscript zero denotes the uniform distribution probabilities, i.e., those valid when all angles are equally probable that is $1 / 11$.

In this study, we use the rotational isomeric state formalism in which each residue is treated as occurring in one or another of several discrete torsion states to obtain the statistics of the chain. $^{21}$

\section{Calculation of a posteriori probabilities}

The statistical weight matrix $\boldsymbol{U}_{i}=\left[u_{\eta \zeta}\right]$ for a given residue pair $i-1$ and $i$ is determined by statistical weights $u_{\eta \zeta}$ corresponding to the energies $E_{\eta \zeta}$ following the work of Flory. ${ }^{21}$

$$
u_{\eta \zeta ; i}=\exp \left(-E_{\eta \zeta ; i} / R T\right)
$$

So, a priori probabilities led us to determine statistical weights that would be the primary quantities for characterizing the partition function. The partition function, $Z$, of the chain of $N$ repeat units is given by

$$
Z=J^{*}\left[\prod_{i=2}^{N} \mathrm{U}_{i}\right] J
$$

where $J^{*}=[11 \ldots 1]$, and $J=$ column[11..1] of order $1 \times 11$ and $11 \times 1$ respectively. We choose all elements of $J^{*}$ as ones to allow all possible states in the first residue as opposed to Flory's notion, $J^{*}=[10 \ldots 0]$, that fixes the first residue's state as the first state.

The probability $p_{\eta ; i}$ that residue $i$ will be in state $\eta$ is estimated as the fraction of the sum of the statistical weights for all configurations for which residue $i$ is in state $\eta$ over partition function $Z{ }^{21}$

$$
p_{\eta ; i}=Z^{-1} J^{*}\left[\prod_{m=2}^{i-1} \boldsymbol{U}_{m}\right] \boldsymbol{U}_{\eta ; i}^{\prime}\left[\prod_{m=i+1}^{N} \boldsymbol{U}_{m}\right] J
$$

Here, $\boldsymbol{U}_{\eta ; i}^{\prime}$ is the matrix obtained by equating the entries of all of its columns to zero except those of column $\eta$. 
Similarly, the joint probability $p_{\eta \zeta ; i-1, i}$ that residue $i-1$ is in state $\eta$ and residue $i$ is in state $\zeta$ simultaneously is given by

$$
p_{\eta \zeta ; i-1, i}=Z^{-1} \boldsymbol{J}^{*}\left[\prod_{m=2}^{i-1} \boldsymbol{U}_{m}\right] \boldsymbol{U}_{\eta \zeta ; i}^{\prime}\left[\prod_{m=i+1}^{N} \boldsymbol{U}_{m}\right] J
$$

where $\boldsymbol{U}_{\eta \zeta ; i}^{\prime}$ is the matrix obtained by vanishing all elements of $\boldsymbol{U}_{i}$ with the exception of $u_{\eta \zeta}$. The transition probability $p_{\eta \mid \zeta}$ is simply the conditional probability that residue $i$ will be in state $\zeta$, given that residue $i-1$ is in state $\eta$ is determined as the quotient of the joint probability, divided by $p_{\eta ; i-1}$.

$$
p_{\eta \mid \zeta}=\mathrm{P}\left(\mathrm{q}_{i}=\zeta \mid \mathrm{q}_{i-1}=\eta\right)=\frac{p_{\eta \zeta ; i-1, i}}{p_{\eta ; i-1}}
$$

\section{The Viterbi algorithm}

Using the Viterbi algorithm is an efficient way of calculating the most probable state sequence. The problem is to find a state sequence $\mathrm{Q}=\mathrm{q}_{1}, \mathrm{q}_{2}, \ldots, \mathrm{q}_{N}$ given an observed sequence $\mathrm{O}=\mathrm{O}_{1}, \mathrm{O}_{2}, \ldots, \mathrm{O}_{N}$ that maximizes $\boldsymbol{P}(\mathrm{Q} \mid \mathrm{O})$. That is $\operatorname{argmax}_{\mathrm{Q}}$ $\boldsymbol{P}(\mathrm{Q} \mid \mathrm{O})$ meaning the given amino acid sequence takes its most probable conformation. Here, argmax denotes the maximum over the full state sequence set $\mathrm{Q}$ of the arguments of the probability function. In the first part of the algorithm, we obtain $\max _{\mathrm{Q}} \boldsymbol{P}(\mathrm{Q} \mid \mathrm{O})$. For arbitrary $t$ and $i$,

$$
\delta_{t}(i)=\max _{\mathrm{q}_{1}, \mathrm{q}_{2}, \ldots, \mathrm{q}_{t-1}} \boldsymbol{P}\left(\mathrm{q}_{1}, \mathrm{q}_{2}, \ldots, \mathrm{q}_{t-1}, \mathrm{q}_{t}=\mathrm{S}_{i} \mathrm{O}_{1}, \mathrm{O}_{2}, \ldots, \mathrm{O}_{t}\right)
$$

is defined. That is the maximum probability of all ways to end in state $\mathrm{S}_{i}$ at $t$ th amino acid and given the amino acids $\mathrm{O}_{1}, \mathrm{O}_{2}, \ldots, \mathrm{O}_{t}$.

$$
\delta_{1}(i)=\boldsymbol{P}\left(\mathrm{q}_{1}=\mathrm{S}_{i} \text { and } \mathrm{O}_{1}\right)
$$

Then, the joint probability of $\mathrm{Q}$ and $\mathrm{O}$ is maximized over all Q as

$$
\max _{\mathrm{Q}} \boldsymbol{P}(\mathrm{Q} \text { and } \mathrm{O})=\max _{i} \delta_{N}(i)
$$

Since the maximum conditional probability is given as

$$
\max _{\mathrm{Q}} \boldsymbol{P}(\mathrm{Q} \mid \mathrm{O})=\max _{\mathrm{Q}} \frac{\boldsymbol{P}(\mathrm{Q} \text { and } \mathrm{O})}{\boldsymbol{P}(\mathrm{O})}
$$

and $\boldsymbol{P}(\mathrm{O})$ is fixed for all $\mathrm{Q}$,

$$
\max _{\mathrm{Q}} \boldsymbol{P}(\mathrm{Q} \mid \mathrm{O})=\max _{\mathrm{Q}} \boldsymbol{P}(\mathrm{Q} \text { and } \mathrm{O})
$$

Hence,

$$
\underset{\mathrm{Q}}{\arg \max } \boldsymbol{P}(\mathrm{Q} \mid \mathrm{O})=\underset{\mathrm{Q}}{\arg \max } \boldsymbol{P}(\mathrm{Q} \text { and } \mathrm{O})
$$

First part of the algorithm

$$
\delta_{1}(i)=\pi_{i} b_{i}\left(\mathrm{O}_{1}\right), \quad 1 \leq i \leq 11
$$

In the initialization step, $\delta_{1}$ is calculated as an array of size eleven holding the probabilities of eleven torsion states for the first amino acid $\mathrm{O}_{1}$ of the given peptide sequence. The probability $\delta_{i}$ is calculated by multiplying the initial probability of the first residue being in state $i$ with the emission probability that state $i$ emits the first residue. The elements of initial probability vector are chosen as 1 so that each state has equal probability. Then, we calculate all $\delta_{t}$ 's inductively.

$$
\delta_{i}(j)=\max _{1 \leq i \leq 11} \delta_{t-1}(i) p_{i j} b_{j}\left(\mathrm{O}_{t}\right), 2 \leq t \leq N, 1 \leq j \leq 11
$$

Here $N$ denotes the number of residues of the given peptide sequence.

Second part of the algorithm. The states $\mathrm{q}_{i}$ 's are obtained by a backtracking technique that searches for the path with the highest probability. We define

$$
\psi_{N}=\underset{1 \leq i \leq 11}{\arg \max } \delta_{N}(i)
$$

and determine the most probable state of the last residue as $\mathrm{q}_{N}=\mathrm{S}_{\psi N}$. The remaining states are then obtained by successive backtracking

$$
\psi_{t}=\underset{1 \leq i \leq 11}{\arg \max } \delta_{i}(i) p_{i \psi_{t+1}}
$$

and then equating $\mathrm{q}_{t}=\mathrm{S}_{\psi t}$.

\section{Conformations of lower probabilities}

Changing preferences during backtracking allows for the generation of conformations with lower probabilities. Thus, for the $t$ th residue, we define

$$
\psi_{t}^{(k)}=\underset{1 \leq i \leq 11}{\arg \max }{ }^{(k)} \delta_{i}(i) p_{i \psi_{t+1}}
$$

where the superscript $(k)$ denotes the $k$ th maximum value. Here, $k=1$ refers to the maximum value, $k=2$ refers second maximum, etc. For each residue, besides the most probable state, we also determine the 2 nd, 3rd, ..., 11th maximum probable state. We use this definition to determine conformations of lower probabilities during the backtracking stage. We refer to this method of generating conformations of lower probabilities as multistep backtracking.

\section{Multistep backtracking}

In this algorithm, we keep the $n$ conformations with highest probability. The multistep backtracking algorithm proceeds as follows:

1. We define

$$
\begin{gathered}
\psi_{N}(j)=\underset{1 \leq i \leq 11}{\arg \max }{ }^{(j)} \delta_{N}(i) \quad 1 \leq j \leq 11 \\
\lambda_{N}(j)=\max _{1 \leq i \leq 11}^{(j)} \delta_{N}(i), \quad 1 \leq j \leq 11
\end{gathered}
$$

where the superscript $(j)$ denotes the $j$ th maximum value. In $\lambda_{N}$, we keep the probabilities of states of the last residue in decreasing order; in $\psi_{N}$ we keep the corresponding states. Then,

2. For the remaining amino acids we define

$$
\begin{gathered}
\psi_{t}(j)=\underset{1 \leq i \leq 11}{\arg \max }{ }^{(j)} \lambda_{t+1}(i) \delta_{t}(j) p_{j \psi_{t+1}(i)}, 1 \leq t<N \\
\lambda_{t}(j)=\max _{1 \leq i \leq 11}^{(j)} \lambda_{t+1}(i) \delta_{t}(j) p_{j \psi_{t+1}(i)}, 1 \leq t<N
\end{gathered}
$$

Here, we calculate the probability of the state sequence of the region going backward from the $N$ th to $t$ th residue by multiplying the probability of the state sequence of the region from the $N$ th to 
$t+1$ st residue by the transition probability from the $t$ th residue to

$t+1$ st residue. The probabilities are stored in decreasing order.

There are a total of $11^{N}$ possible conformations for the sequence of $N$ residues. In order to keep the number of conformations to be considered at a manageable level, we apply some pruning techniques. (i) We remove the state sequences with zero probability of occurring to decrease the complexity. Those are the impossible paths of the HMM. (ii) At each $t$ th step, if the number of stored sequences becomes larger than a specified threshold value $m$, we remove the state sequences those have lowest probabilities and continue with the $m$ sequences with highest probability. The prediction accuracy increases if one keeps the number of stored paths, $m$, large. On the other hand, it is not feasible when considering long peptides (number of residues $>20$ ).

\section{Results and discussion}

Using eqn (18), we calculate the probability distribution of the eleven states for each amino acid in a given peptide sequence. Thus, given a peptide sequence of length $N$, and an amino acid at the $k$ th position, we can estimate the probability of its observed state, with $1 \leq k \leq N$. Prediction accuracy is defined as the fraction of amino acids that are predicted to be in the most probable state. More specifically, it is calculated as the number of correctly predicted residues of type $\mathrm{X}$ in the most probable state sequences using the Viterbi algorithm, over the total number of residues of type X ( p.a. $\left.=n_{\text {correct }} / n_{\text {total }}\right)$. Most populated states are determined as the number of residues of type $\mathrm{X}$ in the most populated state in the coil library over the total number of residues of type $\mathrm{X}$.

We ran 5-fold cross validation for training and testing. The dataset is randomly divided into five subsets, then $4 / 5$ of the fragments are used to obtain training parameters, and $1 / 5$ is used for testing. The procedure is repeated five times, and the prediction accuracy is calculated by averaging the five accuracies. The training parameters, the transition and the emission probabilities, are derived each time by eqn (1)-(7) using the

Table 2 Prediction accuracies for each residue type

\begin{tabular}{lllr}
\hline Amino acid type & Most populated & Viterbi & Improvement \\
\hline ALA & 24.32 & 31.96 & 31.41 \\
GLU & 28.67 & 34.01 & 18.61 \\
LYS & 26.22 & 33.67 & 28.40 \\
MET & 33.72 & 35.21 & 4.40 \\
GLN & 26.77 & 32.39 & 20.99 \\
ARG & 29.72 & 33.2 & 11.71 \\
LEU & 31.66 & 35.51 & 12.18 \\
PHE & 41.14 & 41.09 & 0.00 \\
TYR & 40.65 & 41.68 & 2.54 \\
TRP & 35.58 & 34.69 & 0.00 \\
CYS & 38.71 & 39.41 & 1.81 \\
HIS & 32.36 & 34.74 & 7.35 \\
ASN & 28.24 & 27.9 & 0.00 \\
ASP & 28.96 & 35.66 & 23.15 \\
SER & 28.23 & 35.21 & 24.74 \\
THR & 35.04 & 38.66 & 10.33 \\
ILE & 55.43 & 55.8 & 0.67 \\
VAL & 55.59 & 55.83 & 0.44 \\
GLY & 34.58 & 38.6 & 11.62 \\
PRO & 55.36 & 59.93 & 8.25 \\
Average & 24.32 & 31.96 & 31.41 \\
\hline
\end{tabular}

corresponding training set. Then, the torsion states are predicted using (14)-(18) for each peptide in the corresponding test set. Lastly, the prediction accuracies determined for each test set and the average are calculated.

In Table 2, the prediction accuracies of each amino acid type using the Viterbi algorithm are provided. Simply, we consider the improvement gained by using the Viterbi algorithm comparing to guessing the most populated state in the library. Since the near neighbor effects are taken into account using the Viterbi algorithm, prediction accuracies of some amino acid types are considerably higher. This comparison approach is first applied by Helles and Fonseca ${ }^{18}$ to determine the accuracy of a neural networks method that is used to predict dihedral angle probability distribution for protein coil residues. They compared the accuracies with the probabilities of guessing the most populated state in the data set for each amino acid type. Here, we show the performance of the Viterbi algorithm comparing the accuracies with the most populated state percentages for each amino acid type in a similar manner.

\section{Consideration of less probable states of residues}

It is possible to generalize the prediction accuracy definition as the number of correctly predicted residues of type $\mathrm{X}$ in the $k$ th most probable state sequences using the Viterbi algorithm, over the total number of residues of type $\mathrm{X}$ (p.a. = $\left.n_{\text {correct }}^{(k)} / n_{\text {total }}\right)$. Then, we may add up the prediction accuracies and define the success rate as s.r. $(i)=\sum_{k=0}^{i}$ p.a. $(k)$.

Since we know the probability of each state for each residue in a given peptide sequence, we can tell the probability of seeing the residue in either the most or the next most probable states, and so on. In Fig. 3, we present the improvements when considering not only the most probable states but also the second, third, etc., most probable states. Increasing values of the abscissa values correspond to considering less and less probable states in the prediction.

In Fig. 4, we present the success rates of each amino acid type. The well predicted amino acids are proline, valine, and isoleucine. On the other hand, the small amino acids glycine and asparagine show lower rates of success. It is observed that the prediction method works better on hydrophobic residues. For example the three hydrophobic residues, Pro, Val, and Ile have $\Phi-\Psi$ distributions that are sharply peaked. This decreases the total number of accessible regions in the Ramachandran

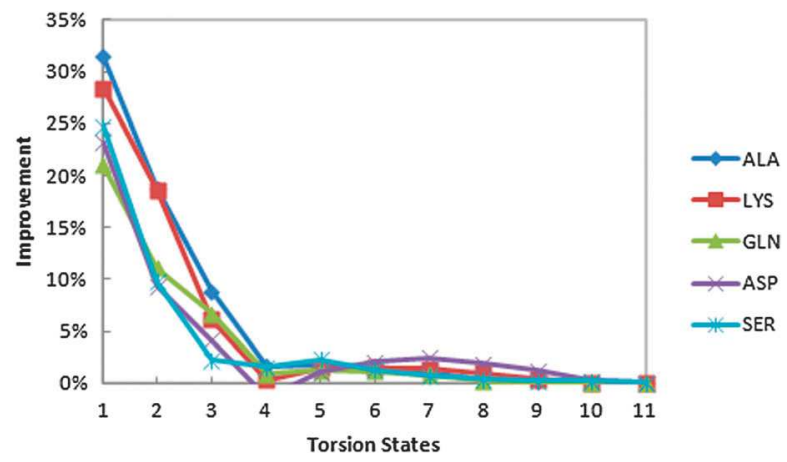

Fig. 3 Percentages of improvements of Ala, Lys, Gln, Asp, and Ser are shown. 


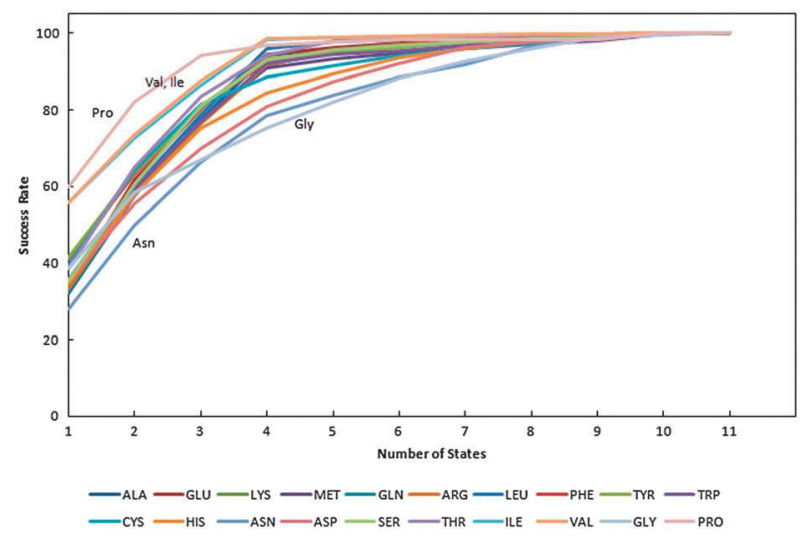

Fig. 4 Success rates of each amino acid types. The $x$ axis shows the number of included states.

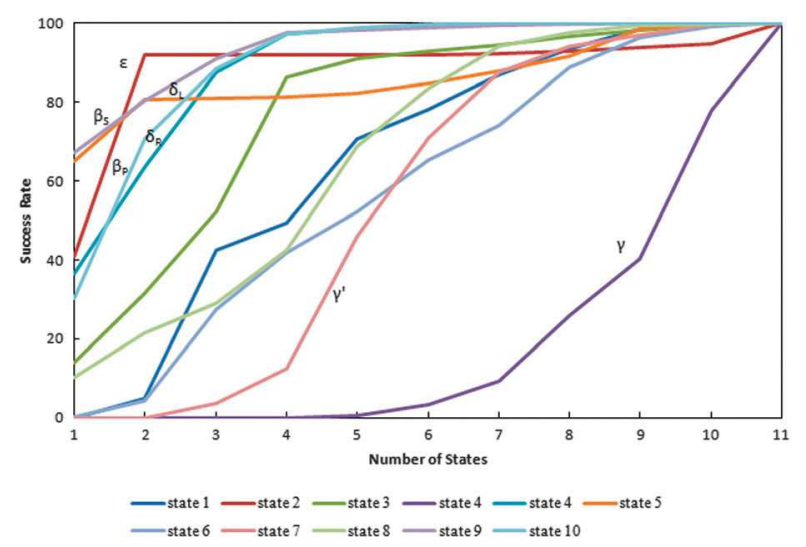

Fig. 5 Success rates of each torsion states. The $x$ axis shows the number of included states.

plot. Majority of the states have zero probability leaving behind only a small region over which the predictions are to be made. The better prediction of hydrophobic residues may be related to this characteristic distribution on the Ramachandran map.

In Fig. 5, we present the success rates of each torsion states. Clearly, prediction of the torsion states that represents the tight turn regions $\left(\gamma\right.$ and $\left.\gamma^{\prime}\right)$ is not successful. This is not surprising because these states are the rarest torsion states in the database. The well predicted states are $\beta_{\mathrm{S}}, \delta_{\mathrm{L}}, \varepsilon, \delta_{\mathrm{R}}$, and $\beta_{\mathrm{p}}$.

\section{Determination of high probability conformations}

The most probable torsion state sequence is predicted using the Viterbi algorithm. The transition and emission probabilities are calculated from the coil database. In the first step of the Viterbi algorithm, the state sequences (Qs) that maximize $\mathrm{P}(\mathrm{Q} \mid \mathrm{O})$ given the peptide sequence $\mathrm{O}$ is determined. Then, in the second step, the sequence $\mathrm{Q}=\mathrm{q}_{1}, \mathrm{q}_{2}, \ldots, \mathrm{q}_{N}$ that has the maximum probability is backtracked where each $\mathrm{q}_{i}$ represents the torsion state of the $i$ th residue. Therefore, after the first step, we may also designate a list of $\mathrm{q}_{i}$ 's for each residue ordered by the probability of occurring. However, our main goal is to find most probable " $n$ " conformations of a given peptide sequence. Hence, using the Viterbi algorithm with multistep backtracking described in the Methods section we derive the most probable " $n$ " conformations of the peptide.

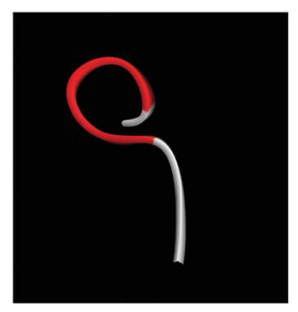

$\beta_{\mathrm{S}^{-}}-\beta_{\mathrm{S}}-\beta_{\mathrm{S}}-\beta_{\mathrm{P}}-\alpha_{\mathrm{L}}-\beta_{\mathrm{P}}-\alpha_{\mathrm{R}}-\alpha_{\mathrm{R}}-\alpha_{\mathrm{R}}-\beta_{\mathrm{S}}$

$\delta_{R}-\beta_{S}-\beta_{S}-\beta_{P}-\alpha_{L}-\beta_{P}-\alpha_{R}-\alpha_{R}-\alpha_{R}-\beta_{S}$

$\beta_{\mathrm{P}}-\beta_{\mathrm{S}}-\beta_{\mathrm{S}}-\beta_{\mathrm{P}}-\alpha_{\mathrm{L}}-\beta_{\mathrm{P}}-\alpha_{\mathrm{R}}-\alpha_{\mathrm{R}}-\alpha_{\mathrm{R}}-\beta_{\mathrm{S}}$

$\alpha_{R}-\beta_{S}-\beta_{S}-\beta_{P}-\alpha_{L}-\beta_{P}-\alpha_{R}-\alpha_{R}-\alpha_{R}-\beta_{S}$

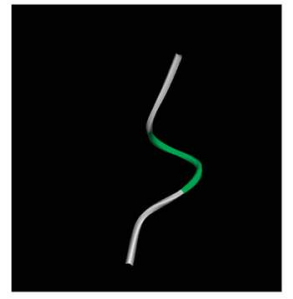

$\beta_{\mathrm{S}}-\beta_{\mathrm{S}}-\beta_{\mathrm{P}}-\beta_{\mathrm{P}}-\beta_{\mathrm{P}}-\alpha_{\mathrm{R}}-\alpha_{\mathrm{R}}-\alpha_{\mathrm{R}}-\beta_{\mathrm{S}}-\beta_{\mathrm{S}}$

$\delta_{\mathrm{R}}-\beta_{\mathrm{S}}-\beta_{\mathrm{P}}-\beta_{\mathrm{P}}-\beta_{\mathrm{P}}-\alpha_{\mathrm{R}}-\alpha_{\mathrm{R}}-\alpha_{\mathrm{R}}-\beta_{\mathrm{S}}-\beta_{\mathrm{S}}$

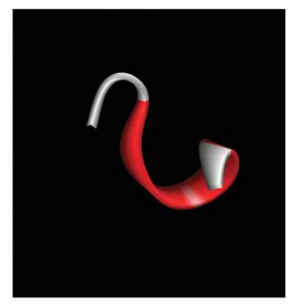

$\beta_{\mathrm{S}}-\beta_{\mathrm{S}}-\beta_{\mathrm{P}}-\delta_{\mathrm{R}}-\alpha_{\mathrm{L}}-\beta_{\mathrm{P}}-\alpha_{\mathrm{R}}-\alpha_{\mathrm{R}}-\alpha_{\mathrm{R}}-\beta_{\mathrm{S}}$ $\delta_{R}-\beta_{S}-\beta_{P}-\delta_{R}-\alpha_{L}-\beta_{P}-\alpha_{R}-\alpha_{R}-\alpha_{R}-\beta_{S}$

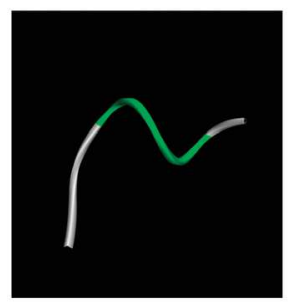

$\beta_{S}-\beta_{S}-\beta_{S}-\delta_{R}-\beta_{P}-\delta_{R}-\alpha_{R}-\alpha_{R}-\alpha_{R}-\beta_{S}$ $\delta_{R}-\beta_{S}-\beta_{S}-\delta_{R}-\beta_{P}-\delta_{R}-\alpha_{R}-\alpha_{R}-\alpha_{R}-\beta_{S}$

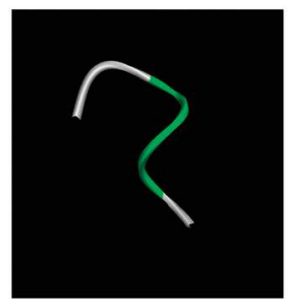

$\beta_{\mathrm{S}}-\beta_{\mathrm{S}}-\beta_{\mathrm{P}}-\alpha_{\mathrm{R}}-\beta_{\mathrm{P}}-\delta_{\mathrm{R}}-\alpha_{\mathrm{R}}-\alpha_{\mathrm{R}}-\alpha_{\mathrm{R}}-\beta_{\mathrm{S}}$

$\delta_{R}-\beta_{S}-\beta_{P}-\alpha_{R}-\beta_{P}-\delta_{R}-\alpha_{R}-\alpha_{R}-\alpha_{R}-\beta_{S}$

Fig. 6 Most probable 12 conformations of the sequence "Gln-ValCys-Ala-Asn-Pro-Glu-Lys-Lys-Trp". From top right to bottom right the state sequences are ordered from the most probable to the 12th most probable conformation. The symbols indicate the torsion states of the residues for the respective conformations. The conformations are determined using the Viterbi algorithm with multistep backtracking with $n=12$ and $m=1000$.

As an example, consider the sequence "GLN-VAL-CYSALA-ASN-PRO-GLU-LYS-LYS-TRP". The most probable twelve conformations are predicted as shown in Fig. 6. It is clear that some conformations repeat although the torsion states of some residues in the state sequences are different. Examination of the states listed below each figure shows that the differences are all in the first residue, which results in the similarity of the conformations. The repeating conformations indicate high entropy for that conformation.

In previous studies, the prediction of the most probable torsion state of a residue has been considered. ${ }^{13,14}$ Helles and 
Fonseca ${ }^{18}$ considered the problem of predicting the probability distribution of the states of the coil residues in a given peptide instead of predicting only the most probable state. As a different approach, we predict the high probable conformations rather than the probabilities of torsion states of the individual residues. Therefore, this is the first study that handles the prediction of high probability conformations.

The predictive capability of a given method depends on the nature of the library used, and the number of states that are used to define the probability space. The non-redundant PDB data set contains information on secondary structures, information on structural motifs and their relation to conformations which may be incorporated into the probability space to be used for predictions. A coil database contains less information, only on residue types and neighbor dependences. An IDP database contains information on the specific structural features of the disordered chains. Comparing the predictions from different data sets is not meaningful. However, just in order to see the relative performances of other methods and ours, we use results of Bystroff et $a l .{ }^{12}$ and Kuang et al. ${ }^{13}$ Both of these studies use the nonredundant PDB data base. Bystroff et al. ${ }^{12}$ used eleven states using a different definition from our torsion state definition and proposed an HMM model based on a library of sequencestructure motifs rather than the coil regions. Kuang et al. ${ }^{13}$ defined four states (A, B, G, E) and used an SVM method based on protein sequence profiles. Since these methods use different torsion state definitions, Kuang et al. ${ }^{13}$ suggested to group the states given by Bystroff et al. ${ }^{12}$ into four states $\left(\mathrm{A}^{\prime}, \mathrm{B}^{\prime}, \mathrm{G}^{\prime}, \mathrm{E}^{\prime}\right)$ for comparison. The grouped states are only approximately equivalent. Then, the prediction accuracies are determined as $74 \%$ for HMMSTR and $77.3 \%$ for SVM methods. We follow the same idea and group our eleven torsion states into four states as $\left(\mathrm{A}^{\prime \prime}=\right.$ $\alpha_{\mathrm{R}}+\delta_{\mathrm{R}}, \mathrm{B}^{\prime \prime}=\beta_{\mathrm{P}}+\beta_{\mathrm{S}}+\varepsilon^{\prime}+\gamma^{\prime}+\zeta, \mathrm{G}^{\prime \prime}=\alpha_{\mathrm{L}}+\delta_{\mathrm{L}}+\gamma$, $\mathrm{E}^{\prime \prime}=\varepsilon$ ). Then, the prediction accuracy of the Viterbi algorithm is $59.2 \%$ for predicting the most probable state of a residue in a given peptide sequence of the coil library. The additional $15 \%$ improvement may approximately be ascribed to the additional structural information content in the non-redundant PDB data set over that of the coil dataset.

Predicting highly probable conformations of a given peptide sequence is an important issue in targeted drug design. To be effective, a short peptide must match a given region of the surface of a protein, producing a change in the activities of proteins that can affect certain diseases. ${ }^{24}$ The conformations of the peptide that fulfil this constraint have to be among the most probable ones for maximum stability.

Another important concept is the intrinsically disordered proteins those do not naturally adopt a unique three dimensional structure. Therefore, their structure ranges from the totally random coil to molten globular. ${ }^{27,29}$ Use of a torsion state library for IDP's and the present computational model will lead to the prediction of high probability conformations of disordered proteins.

\section{Conclusion}

Predicting " $n$ " high probability conformations of a given peptide problem is discussed. The proposed method is based on the RIS model and the Viterbi algorithm with multistep backtracking. The necessary parameters of the HMM are derived from a coil library depending on the residue types and the near neighbour effects.

While using the Viterbi algorithm the long-range interactions are not included and may be essential for the state prediction. One may introduce second, or third neighbor effects that also would improve the prediction accuracies, since the coil regions are not exactly random. ${ }^{30}$ Also, the scarcity of data for some torsion states in the coil library leads to difficulty in prediction.

The present paper discusses the prediction of conformations of random coiled proteins from a primary sequence. We showed that for certain amino acid types, introduction of residue type and neighbor dependence improved the predictions of conformations of proteins in the coil state. Thus, we showed that the Flory Isolated Pair Hypothesis does not hold in general.

\section{Notes and references}

1 G. N. Ramachandran, C. Ramakrishnan and V. Sasisekharan, J. Mol. Biol., 1963, 7, 95-99.

2 F. Avbelj and R. L. Baldwin, Proc. Natl. Acad. Sci. U. S. A., 2004, 101, 10967-10972.

3 F. Avbelj, S. G. Grdadolnik, J. Grdadolnik and R. L. Baldwin, Proc. Natl. Acad. Sci. U. S. A., 2006, 103, 1272-1277.

4 O. Engin, M. Sayar and B. Erman, Phys. Biol., 2009, 6, 016001.

5 J. F. Gibrat, B. Robson and J. Garnier, Biochemistry, 1991, 30, $1578-1586$.

6 O. Keskin, D. Yuret, A. Gursoy, M. Turkay and B. Erman, Proteins: Struct., Funct., Bioinf., 2004, 55, 992-998.

7 L. Ormeci, A. Gursoy, G. Tunca and B. Erman, Proteins: Struct., Funct., Genet., 2007, 66, 29-40.

8 R. V. Pappu, R. Srinivasan and G. D. Rose, Proc. Natl. Acad. Sci. U. S. A., 2000, 97, 12565-12570.

9 V. Pappu, R. Srinivasan and G. D. Rose, Proc. Natl. Acad. Sci. U. S. A., 2000, 97, 12565-12570.

10 M. H. Zaman, M. Y. Shen, R. S. Berry, K. F. Freed and T. R. Sosnick, J. Mol. Biol., 2003, 331, 693-711.

11 N. C. Fitzkee, P. J. Fleming and G. D. Rose, Proteins: Struct., Funct., Genet., 2005, 58, 852-854.

12 C. Bystroff, V. Thorsson and D. Baker, J. Mol. Biol., 2000, 301, 173-190.

13 R. Kuang, C. S. Leslie and A.-S. Yang, Bioinformatics, 2004, 20, $1612-1621$.

14 O. Zimmermann and U. H. E. Hansmann, Bioinformatics, 2006, 22, 3009-3015.

15 S. C. Lovell, I. W. Davis, W. B. Arendall, P. I. W. de Bakker, J. M. Word, M. G. Prisant, J. S. Richardson and D. C. Richardson, Proteins: Struct., Funct., Bioinf., 2003, 50, 437-450.

16 D. Shortle, Protein Sci., 2002, 11, 18-26.

17 G. Xin, G. Jihong, D. Qiwen and Z. Shuigeng, Bioinformatics and Biomedical Engineering (iCBBE), 2010 4th International Conference on, 2010.

18 G. Helles and R. Fonseca, BMC Bioinf., 2009, 10, 338.

19 W. Boomsma, K. V. Mardia, C. C. Taylor, J. Ferkinghoff-Borg, A. Krogh and T. Hamelryck, Proc. Natl. Acad. Sci. U. S. A., 2008, 105, 8932-8937.

20 Z. Wang, F. Zhao, J. Peng and J. Xu, Proteomics, 2011, 11, 3786-3792.

21 P. J. Flory, Statistical mechanics of chain molecules, Interscience Publishers, New York, 1969.

22 P. A. Karplus, Protein Sci., 1996, 5, 1406-1420.

23 B. Unal, A. Gursoy and B. Erman, PLoS One, 2010, 5, e10926.

24 E. B. Unal, A. Gursoy and B. Erman, Phys. Biol., 2009, 6, 036014.

25 G. W. Daughdrill, S. Kashtanov, A. Stancik, S. E. Hill, G. Helms, M. Muschol, V. Receveur-Brechot and F. M. Ytreberg, Mol. BioSyst., 2012, 8, 308-319.

26 W. J. Ewens and G. R. Grant, Statistical methods in bioinformatics: an introduction, Springer, New York, 2001.

27 R. Schweitzer-Stenner, Mol. BioSyst., 2012, 8, 122-133.

28 M. M. Babu, Mol. BioSyst., 2012, 8, 21.

29 V. N. Uversky, Protein Sci., 2002, 11, 739-756.

30 N. C. Fitzkee and G. D. Rose, Proc. Natl. Acad. Sci. U. S. A., 2004, 101, 12497-12502. 